\title{
Optimal allocation of Sen Transformer for active power loss reduction
}

\begin{abstract}
Reduction of active power loss is one of the main functions that are carried in power system control centers. The main purpose of transmission systems is efficiently handling power from generation stations to load centers. Yet, a considerable amount of power is lost in transmission network components. As a corrective action, power flow redistribution in transmission lines using appropriate power flow control devices is useful to increase the transmission efficiency. However, the inserted power flow control devices themselves consume power. Thus, if not optimally sited, their own power loss may overcome the loss reduction which is obtained by their action, and as a consequence may increase the power loss further. Optimal allocation of these devices is critical to ensure fulfillment of the proposed goals. In this work, optimally placed Sen Transformer (ST) is suggested for power loss reduction considering security constraints. ST is modelled under MATLAB/SIMULINK, and optimally located using active power loss sensitivity index. The method is validated using IEEE-6 bus system and the results are encouraging.
\end{abstract}

Keyword: Active power loss; Loss sensitivity; Optimal allocation; Power flow control; Sen Transformer 